\title{
Design of a Porous Cored Hexagonal Photonic Crystal Fiber Based Optical Sensor With High Relative Sensitivity for Lower Operating Wavelength
}

\author{
Shuvo SEN ${ }^{1}$, Sawrab CHOWDHURY ${ }^{1}$, Kawsar AHMED ${ }^{1,2^{*}}$, and \\ Sayed ASADUZZAMAN ${ }^{2}$ \\ ${ }^{1}$ Department of Information and Communication Technology, Mawlana Bhashani Science and Technology University, \\ Santosh, Tangail-1902, Bangladesh \\ ${ }^{2}$ Group of Biophotomatix, Bangladesh
}

*Corresponding author: Kawsar AHMEDＥmail: kawsar.ict@mbstu.ac.bd and k.ahmed.bd@ieee.org

\begin{abstract}
In this article, highly sensitive and low confinement loss enriching micro structured photonic crystal fiber (PCF) has been suggested as an optical sensor. The proposed PCF is porous cored hexagonal (P-HPCF) where cladding contains five layers with circular air holes and core vicinity is formed by two layered elliptical air holes. Two fundamental propagation characteristics such as the relative sensitivity and confinement loss of the proposed P-HPCF have been numerically scrutinized by the full vectorial finite element method (FEM) simulation procedure. The optimized values are modified with different geometrical parameters like diameters of circular or elliptical air holes, pitches of the core, and cladding region over a spacious assortment of wavelength from $0.8 \mu \mathrm{m}$ to $1.8 \mu \mathrm{m}$. All pretending results exhibit that the relative sensitivity is enlarged according to decrement of wavelength of the transmission band $(\mathrm{O}+\mathrm{E}+\mathrm{S}+\mathrm{C}+\mathrm{L}+\mathrm{U})$. In addition, all useable liquids reveal the maximum sensitivity of $57.00 \%, 57.18 \%$, and $57.27 \%$ for $n=1.33,1.354$, and 1.366 respectively by lower band. Moreover, effective area, nonlinear coefficient, frequency, propagation constant, total electric energy, total magnetic energy, and wave number in free space of the proposed P-HPCF have been reported recently.
\end{abstract}

Keywords: Optical sensor; relative sensitivity; porous core PCF; confinement loss; transmission band; effective area

Citation: Shuvo SEN, Sawrab CHOWDHURY, Kawsar AHMED, and Sayed ASADUZZAMAN, "Design of a Porous Cored Hexagonal Photonic Crystal Fiber Based Optical Sensor With High Relative Sensitivity for Lower Operating Wavelength," Photonic Sensors, 2017, 7(1): 55-65.

\section{Introduction}

Photonic crystal fiber (PCF) is a novel invention of optical fiber technology which has ensured an immense advancement in telecom and nonlinear devices applications [1]. It consists of an arbitrary order [2] of tiny air holes which trip along the entire length of the fiber or periodic array [3]. Indexed guiding (IG) and photonic band gap (PBG) are two principal types of photonic crystal fibers according to propagation characteristics of light. In index guiding (IG), PCF has sustained superior refractive index than the cladding region with a solid core $[4$, 5]. In addition, light is instructed by photonic band gap principle with a large air core in PBG PCF [6]. In 1996, Knight et al. [7] first fabricated a

Received: 13 September 2016 / Revised: 1 November 2016

(C) The Author(s) 2016. This article is published with open access at Springerlink.com

DOI: $10.1007 / \mathrm{s} 13320-016-0384-\mathrm{y}$

Article type: Regular 
hexagonal-PCF structure, and this procedure was modernized day by day to obtain better guiding properties. At the present time, researchers attained better guiding properties to utilize an octagonal [2], decagonal [8], honeycomb cladding [9], circular, and hybrid [10] designed PCF structure. Due to the promotion of fabrication technologies, high sensitivity [11], high birefringence [12], ultraflattened dispersion [13], and high nonlinear effect [14] are achieved by scheming of PCF with altering the air hole diameters, pitch, and shape.

PCF can subdue many restrictions of conventional fibers by following a few supplemental features in design such as values of pitch, air-hole diameter, and number of rings both the core and cladding. Photonic crystal fibers become extremely admired due to low cost, small size, robustness, enhanced design freedom, and flexibility. It has been attracted much concentration for its improvable performance and enormous diversity of applications [15]. PCF can be accustomed like as switches [16], electro-optical modulators [17, 18], filters [19], and polarization converters [20]. PCFs are guaranteed a revolutionary improvement in spectroscopy [21], super continuum generation [22], and optical communication [1] applications due to its singular characteristic. Optical sensors are updated day by day with the advancement of technology and a unique geometrical structure.

Refractive index (RI) sensors [23], gas sensing [4], liquid sensors [24], pressure sensors [25], temperature sensors [26], mechanical sensors [27], chemical sensors, and $\mathrm{P}^{\mathrm{H}}$ sensors [28] are the broad range implementation of PCF sensors. According to fascinate attraction in chemical and biomedical [28, 29] applications, the evanescent wave based PCF sensors are expanding quickly. The evanescent field of PCFs is prevalently entangled in gas sensing with chemical and bio sensing [28-30] and different index materials [23]. In addition, the bacteria sensor [30] is accustomed with the evanescent wave based PCF sensor. To subdue the safety problem in the industrial adaptation [31] particularly for perception of toxic and flammable chemicals, noble sensitive chemical (liquid and gas) sensors are performed a significant rule. PCF based sensors are also attracted much concentration of researchers in environmental and safety monitoring $[23,31]$ applications.

In order to maximize the relative sensitivity and reduce confinement loss at adequate degree in liquid [24] or chemical sensing [28] applications, the researchers has been reported plenty of articles by replacing distinctive geometric parameters of the PCF. Lee and Asher [28] sensed echanical sensors [27], chemical sensors, and $\mathrm{P}^{\mathrm{H}}$ level and ionic strength by using a PCF based chemical sensor. In 2001, Hansen et al. [32] reported a highly birefringence index guiding PCF which depicted a little bit higher birefringence contrast with conventional fibers. A hybrid-PCF structure is constituted by three rings cladding of circular air holes and micro structured core with elliptical air holes which depicts high birefringence, and sensitivity and low confinement loss was proposed by Asaduzzaman et al. [10]. In [33], it was recommended that lower confinement loss, dispersion, and nonlinear effect are pointed out by a nanostructure index guiding PCF.

In this article, a porous cored hexagonal photonic crystal fiber (P-HPCF) has been suggested which reveals high sensitivity and low confinement loss for three thermo optical coefficient like water $(n=1.330)$, ethanol $(n=1.354), \quad$ and benzene $(n=1.366)$. Our proposed P-HPCF is formed by five layered circular air holes based cladding and two layered porous cored with elliptical air holes. The geometrical parameters have been diverse with both the optimized structure core and cladding territory of proposed P-HPCF and improved the relative sensitivity with low confinement loss at the same time compared with prior PCFs [10, 34, 35].

\section{Geometries of the proposed P-HPCF}

The schematic cross sectional scenery of the proposed P-HPCF is presented in Fig. 1. In addition, 
this figure is plainly outlined the entire geometrical structure of the PCF. The proposed P-HPCF structure is hexagonal where the core is porous shaped. In the cladding region, the vertices of the attaching air cavities contain a $60^{\circ}$ angle that forms hexagonal shape.

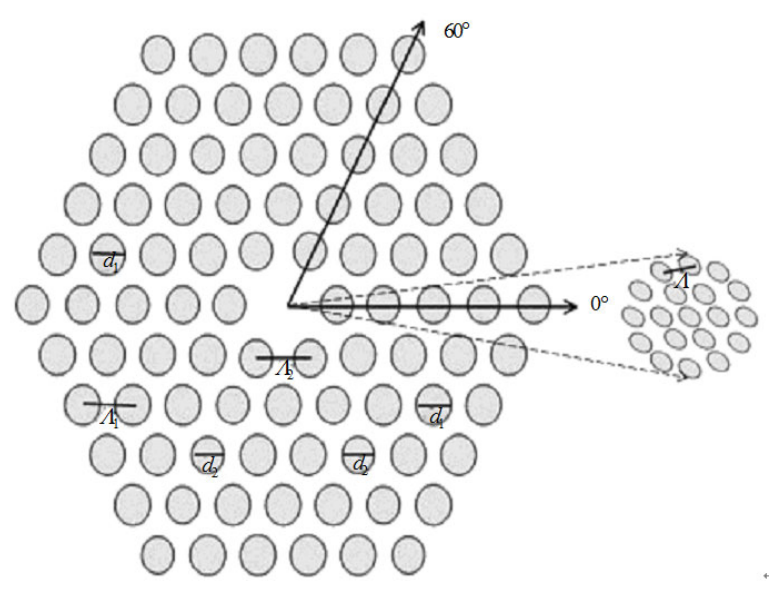

Fig. 1 Schematic cross sectional view of the proposed porous cored hexagonal photonic crystal fiber (P-HPCF) and enlarged view of its 2-layer porous core.

The first, second, third, fourth and fifth layers are restrained with $6,12,18,24$, and 30 air cavities, respectively. Pitch can be determined by the distance between the two air holes (at cladding) for entire types of fibers and procedures which are denoted by $\Lambda_{1}$ and $\Lambda_{2}$. The diameters of air cavities in every layer of cladding are presented by $d_{1}$ and $d_{2}$. At the core territory, the vertices of the adjoining 6 elliptical air holes of the first layer contain a $60^{\circ}$ angle which is arranged in a porous shape. Due to the second layers, the vertices of the adjoining elliptical air holes contain a $30^{\circ}$ angle, and the number of elliptical air holes is 12. All elliptical air holes in the core region are arranged in a $135^{\circ}$ angle perspective to original position. As a result, the proposed PCF design cannot be collapsed. Utilizing the Sellmeier equation's [36] refractive index (n) and absolute silica as background, material has been chosen for all kinds of fibers. The diameter and pitch of the complementary elliptical air holes of porous cored are marked by $d_{c 1}, d_{c 2}$, and $\Lambda$, respectively. All supplementary elliptical air holes are filled with three different thermo optic coefficients like water $(n=1.33)$, ethanol $(n=1.354)$, and benzene $(n=1.366)$ respectively at the core territory.

\section{Synopsis of numerical method}

All numerical results of the proposed P-HPCF can be examined by applying the full vectorial finite element method (FEM). To model and improve photonic ingredients or devices for engineers, perfectly matched layers (PML) boundary condition is referred as the most effective numerical approach [37-41]. According to the boundary condition, PML is fixed at $10 \%$ of the entire diameter of the proposed P-HPCF [42]. The thickness of circular PML is chosen as $1.1 \mu \mathrm{m}$. The propagation characteristics such as relative sensitivity and the confinement loss are obtained from Maxwell's equations [43].

The power of light can be infiltrated of the cladding territory from the core because the finite number of air cavities of a photonic crystal fiber is considered as the confinement loss which is denoted by $L_{c}$. Kaneshima et al. [44] expressed the confinement loss $(\mathrm{dB} / \mathrm{m})$ by

$$
L_{c}=8.68 k_{0} \operatorname{Im}\left[n_{\text {eff }}\right]
$$

where $k_{0}=2 \pi \lambda ; \lambda$ is the wavelength of light, and $\operatorname{Im}\left[n_{\text {eff }}\right]$ is known as the imaginary part of the refractive index. The relative sensitivity coefficient $r$ can be computed by the mutual action between light and the analyte [45]. To estimate the relative sensitivity, coefficient $r$ is enforced by the following equation:

$$
r=\frac{n_{r}}{n_{\text {eff }}} f
$$

where $n_{r}$ and $n_{\text {eff }}$ are marked as the refractive index of sensed material within the air cavities and the modal effective index. The fraction of air cavity power and the absolute power percentage can be calculated through (3) and represented by $f$ :

$$
f=\frac{\int_{\text {sample }} \operatorname{Re}\left(E_{x} H_{y}-E_{y} H_{x}\right) d x d y}{\int_{\text {total }} \operatorname{Re}\left(E_{x} H_{y}-E_{y} H_{x}\right) d x d y} \times 100
$$

where $E_{x}$ and $H_{x}$ represent the diagonal electric field 
and magnetic field. $E_{y}$ and $H_{y}$ represent the longitudinal electric field and magnetic field. The FEM is implemented to procure the mode field pattern $E_{x}, H_{x}, E_{y}, H_{y}$, and formal effective index $n_{\text {eff }}$ correspondingly. The effective area $A_{\text {eff }}$ [46] of a PCF can be calculated by the following equation:

$$
A_{\text {eff }}=\frac{\left(\iint|E|^{2} d x d y\right)^{2}}{\iint|E|^{4} d x d y}
$$

where optical power is denoted by $E$. A small effective area is caused for high optical power density which also indicates the nonlinear effect. Equation (5) is utilized to calculate the nonlinear coefficient $(\gamma)$ [46]

$$
\gamma=\frac{n_{2} \omega}{C A_{\text {eff }}}=\frac{n_{2} 2 \pi}{\lambda A_{\text {eff }}}[\omega=2 n \pi \text { and } C=n \lambda]
$$

where $C, n$, and $\lambda$ present the velocity, frequency, and wavelength of light, respectively. The nonlinearindex coefficient $n_{2}$ in the nonlinear part of the refractive index is $\delta_{n}=n_{2}|E|^{2}$.

\section{Numerical results and discussion}

In this section, the guiding properties of the reported P-HPCF have been examined in agreement with assorted geometrical parameters. The complementary elliptical air cavities of core region is satiated by three thermo optic coefficients such as water $(n=1.330)$, ethanol $(n=1.354)$, and benzene $(n=1.366)$. Numerical investigation into the P-HPCF has been carried out at a wide range of wavelength from $0.8 \mu \mathrm{m}$ to $1.8 \mu \mathrm{m}$. The extensive pretending procedure has been performed by COMSOL Multiphysics 4.2 versions. The convergence error of the proposed P-HPCF is very low which is approximately at $1.50 \times 10^{-8} \%$ for the optimum parameters.

The electric field distributions for (a) $x$ polarization and (b) $y$-polarization at an activation wavelength of $1.33 \mu \mathrm{m}$ have been presented in Fig. 2 . The mode field is firmly restricted in the core vicinity which increases the sensitivity of the proposed P-HPCF. As a result, the leakage loss of the fiber is very low.

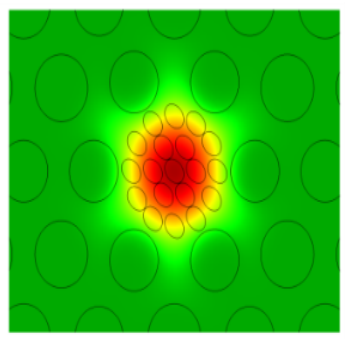

(a)

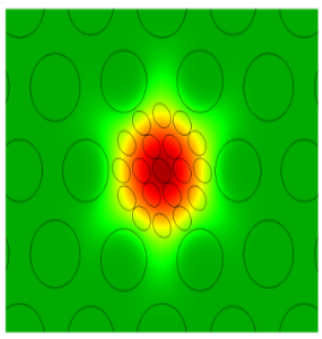

(b)
Fig. 2 Optical field distribution of the proposed P-HPCF: (a) $x$-polarization and (b) $y$-polarization for ethanol $(n=1.354)$ at $\lambda=1.33 \mu \mathrm{m}$.

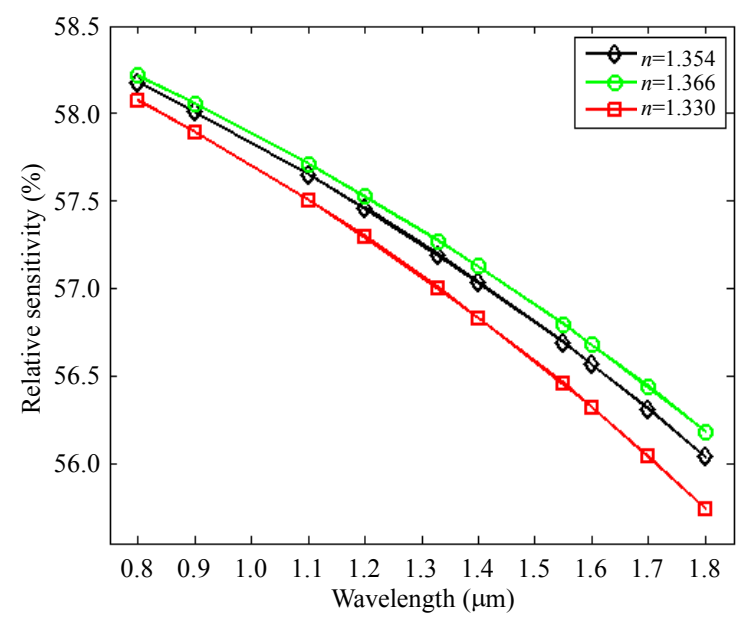

Fig. 3 Variation of the relative sensitivity versus wavelength of the proposed P-HPCF when all parameters are kept optimum.

Figure 3 reveals the influence on relative sensitivity of the proposed P-HPCF for the optimum values. This figure also indicates that the sensitivity is increased with the decrement of wavelength. The proposed P-HPCF shows conflict result compared with those shown in $[10,34,35]$ due to the fact that those articles achieved high sensitivity with the increment of wavelength. The relative sensitivity of proposed P-HPCF is $57 \%, 57.18 \%$, and $57.27 \%$, respectively for three liquids like water $(n=1.330)$, ethanol $(n=1.354)$ and benzene $(n=1.366)$ at $1.33 \mu \mathrm{m}$ wavelength. Besides, confinement loss of the fiber is $2.15 \times 10^{-10} \mathrm{~dB} / \mathrm{m}, \quad 1.11 \times 10^{-11} \mathrm{~dB} / \mathrm{m}, \quad$ and $1.97 \times 10^{-11} \mathrm{~dB} / \mathrm{m}$, respectively in the same condition. In addition, the degree of freedom is $d_{1}=1.80 \mu \mathrm{m}$, $d_{2}=1.96 \mu \mathrm{m}, \Lambda_{1}=2.8 \mu \mathrm{m}, \Lambda_{2}=3 \mu \mathrm{m}, d_{c 1}=0.40 \mu \mathrm{m}, d_{c 2}=$ $0.30 \mu \mathrm{m}$, and $\Lambda=0.80 \mu \mathrm{m}$ for the optimum structure. The optimization of the proposed P-HPCF has done following a simple technique [35]. The first cladding region is optimized. 


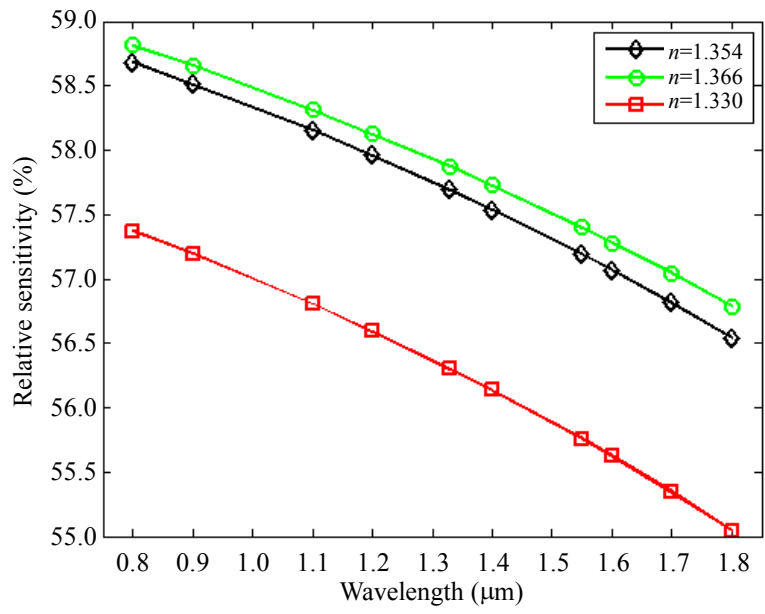

Fig. 4 Variation of the relative sensitivity versus wavelength of the P-HPCF changing the cladding diameter of $+4 \%$ compared with the optimum value when other parameters are kept constant.

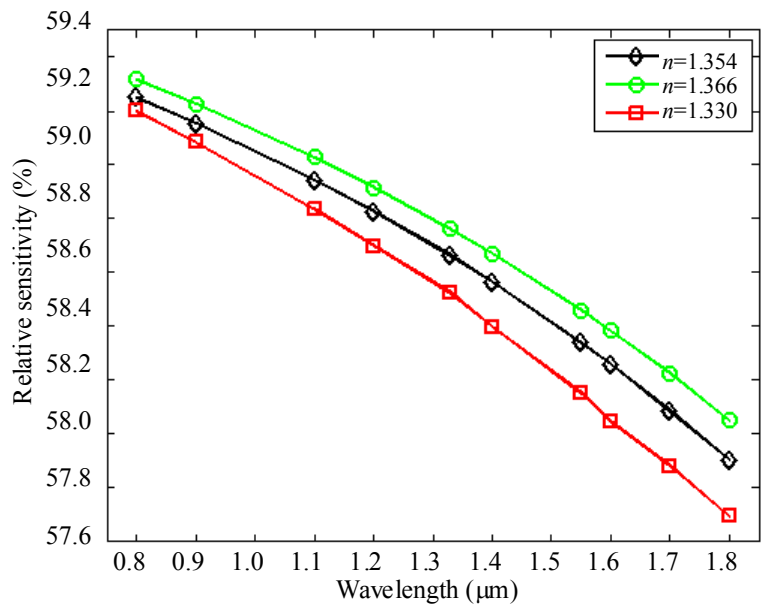

Fig. 5 Variation of the relative sensitivity versus wavelength of the P-HPCF changing the cladding diameter of $+8 \%$ compared with the value when other parameters are kept constant.

Figure 4 demonstrates the effect on the relative sensitivity to fluctuate the cladding air-hole diameters $\left(d_{1}\right.$ and $\left.d_{2}\right)$ of $+4 \%$ with the optimum values when other parameters are kept same for the proposed P-HPCF. The sensitivity of $56.52 \%$, $57.82 \%$, and $57.96 \%$ and the confinement loss of $3.13 \times 10^{-10} \mathrm{~dB} / \mathrm{m}, \quad 5.95 \times 10^{-10} \mathrm{~dB} / \mathrm{m}, \quad$ and $2.5 \times 10^{-10} \mathrm{~dB} / \mathrm{m}$ correspondingly have gained at $1.33 \mu \mathrm{m}$ wavelength. Now it is clearly visualized that sensitivity has increased for two liquids except water $(n=1.330)$ compared with the optimum value.

Figure 5 shows the impact on the relative sensitivity, $+8 \%$ variation with the optimum values of cladding air-hole diameters $\left(d_{1}\right.$ and $\left.d_{2}\right)$ when other parameters have remained constant for the proposed P-HPCF. The sensitivity of $58.37 \%$, $58.51 \%$, and $58.59 \%$ accordingly has been reported for three analytes at $1.33 \mu \mathrm{m}$ wavelength which is little bit high compared with the optimum value. Besides, the confinement loss of the order of $1.92 \times 10^{-9} \mathrm{~dB} / \mathrm{m}, \quad 5.80 \times 10^{-11} \mathrm{~dB} / \mathrm{m}, \quad$ and $4.79 \times 10^{-11} \mathrm{~dB} / \mathrm{m}$ accordingly has also been achieved for desired liquids but it is also high compared with the optimum value. Same results have been found for $+12 \%$ increment of cladding diameters which is shown in Fig. 6 .

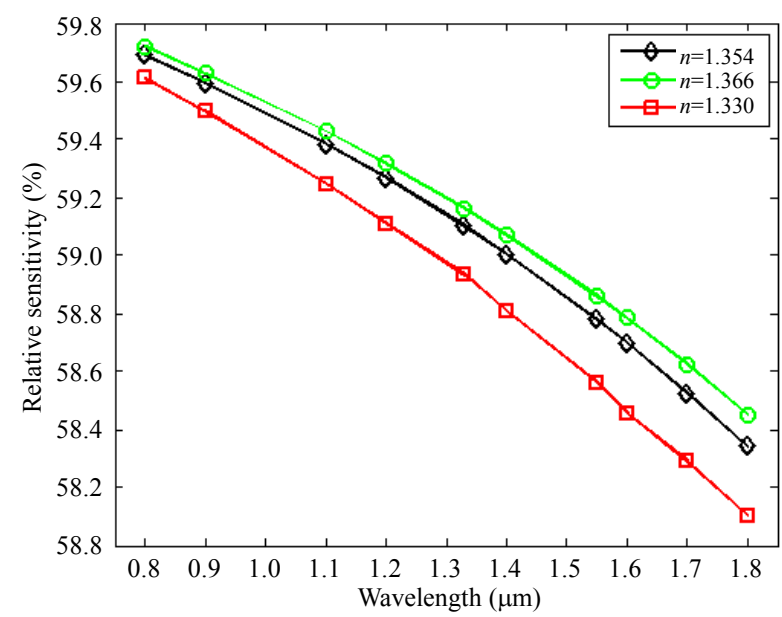

Fig. 6 Variation of the relative sensitivity versus wavelength of the P-HPCF changing the cladding diameter of $+12 \%$ compared with the optimum value when other parameters are kept constant.

All examined results are shown in Figs. 4, 5, and 6. It has been signified that relative sensitivity of the proposed P-HPCF is enlarged when we increase the values of $d_{1}$ and $d_{2}$ with the optimum value. But to avoid fabrication complexity, $d_{1}=1.80 \mu \mathrm{m}$ and $d_{2}=$ $1.96 \mu \mathrm{m}$ have been selected as an optimum value in the cladding region.

Figure 7 demonstrates the influence on the relative sensitivity for decreasing the cladding airhole diameters $\left(d_{1}\right.$ and $\left.d_{2}\right)$ of $-4 \%$ with the optimum values when other parameters are remained the same for the proposed P-HPCF. The sensitivity of $56.23 \%$, $56.43 \%$, and $56.52 \%$ and confinement loss of $1.92 \times 10^{-9} \mathrm{~dB} / \mathrm{m}, \quad 5.80 \times 10^{-11} \mathrm{~dB} / \mathrm{m}, \quad$ and $4.79 \times 10^{-11} \mathrm{~dB} / \mathrm{m}$ respectively have been achieved at $1.33 \mu \mathrm{m}$ wavelength. Now it is clearly visualized 
that sensitivity has decreased due to $-4 \%$ variations with the optimum value for three liquids. Similar results have been found for $-8 \%$ decrement of cladding diameters in Fig. 8 .

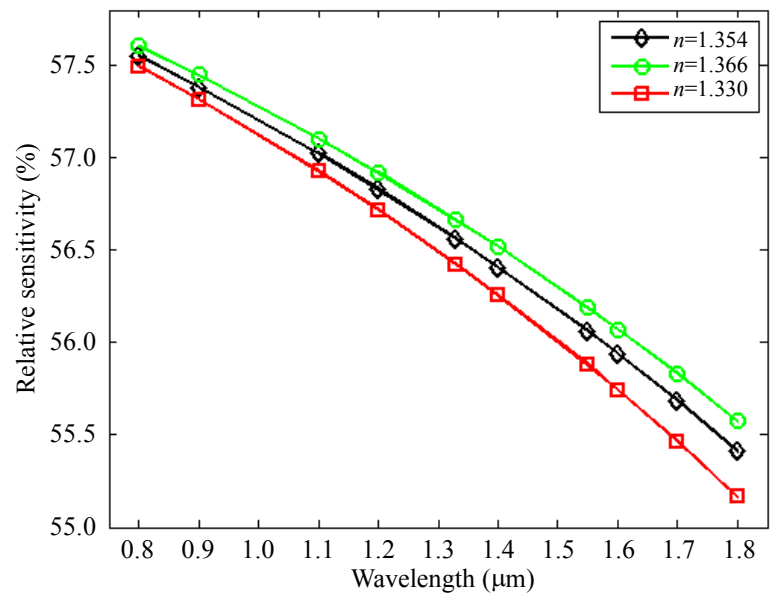

Fig. 7 Variation of the relative sensitivity versus wavelength of the P-HPCF changing the cladding diameter of $-4 \%$ compared with the optimum value when other parameters are kept constant.

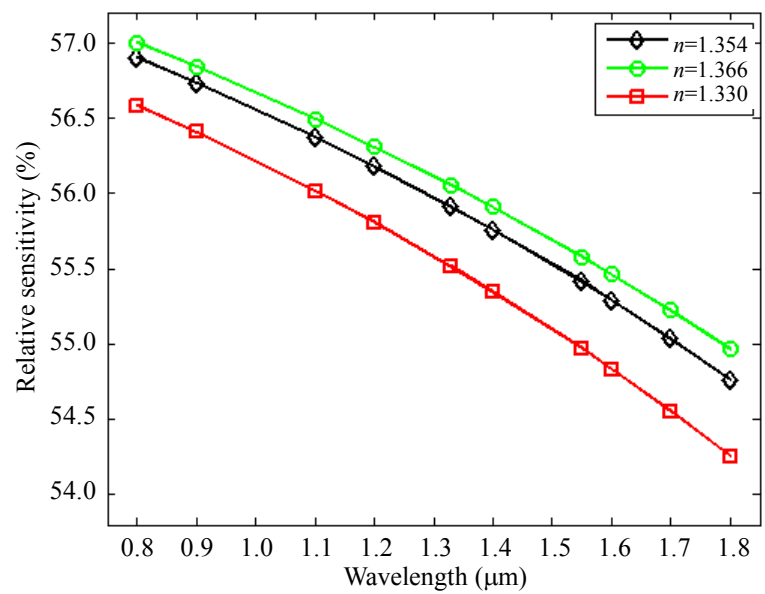

Fig. 8 Variation of the relative sensitivity versus wavelength of the P-HPCF changing the cladding diameter of $-8 \%$ compared with the optimum value when other parameters are kept constant.

After completing all investigations from Figs. 7 and 8 , it has been indicated that propagation characteristics like sensitivity of the reported PHPCF is diminished due to a decrease in the optimum values of cladding air-hole diameters $\left(d_{1}\right.$ and $d_{2}$ ). Next the core territory is optimized.

Figure 9 represents the effect on the relative sensitivity of the proposed P-HPCF for interchanging the air-hole diameters $\left(d_{c 1}\right.$ and $\left.d_{c 2}\right)$ of core vicinity with the optimum values. After completing this procedure, the sensitivity and confinement loss are turned into little bit changes. The sensitivity of $56.90 \%, 57.19 \%$, and $57.14 \%$ and confinement loss of $2.79 \times 10^{-10} \mathrm{~dB} / \mathrm{m}$, $4.39 \times 10^{-10} \mathrm{~dB} / \mathrm{m}$, and $1.81 \times 10^{-11} \mathrm{~dB} / \mathrm{m}$ correspondingly are obtained at $1.33 \mu \mathrm{m}$ wavelength for three thermo optic coefficients. Since the relative sensitivity is slightly decreased with exchanging core air-hole diameters, $d_{c 1}=0.40 \mu \mathrm{m}$ and $d_{c 2}=$ $0.30 \mu \mathrm{m}$ are chosen as the optimum values of airhole diameters in the core region.

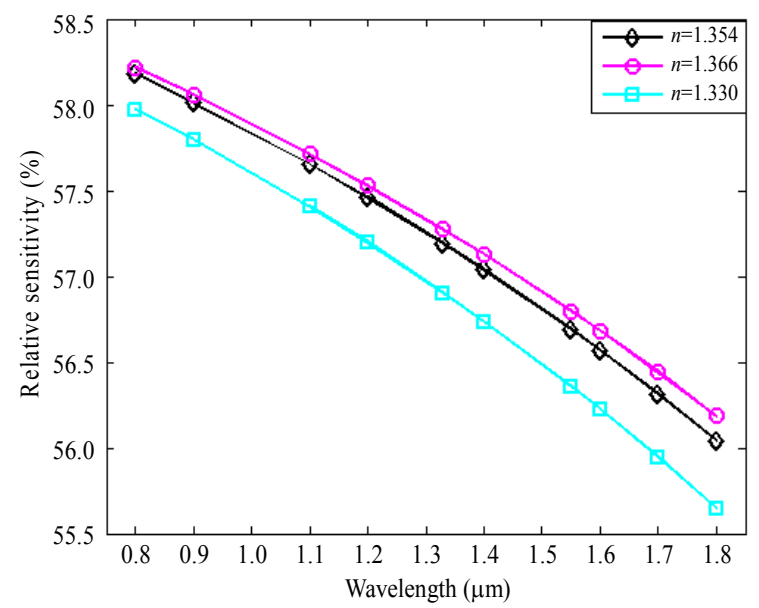

Fig. 9 Variation of the relative sensitivity versus wavelength of the P-HPCF interchanging the core vicinity air-hole diameters when other parameters are kept constant.

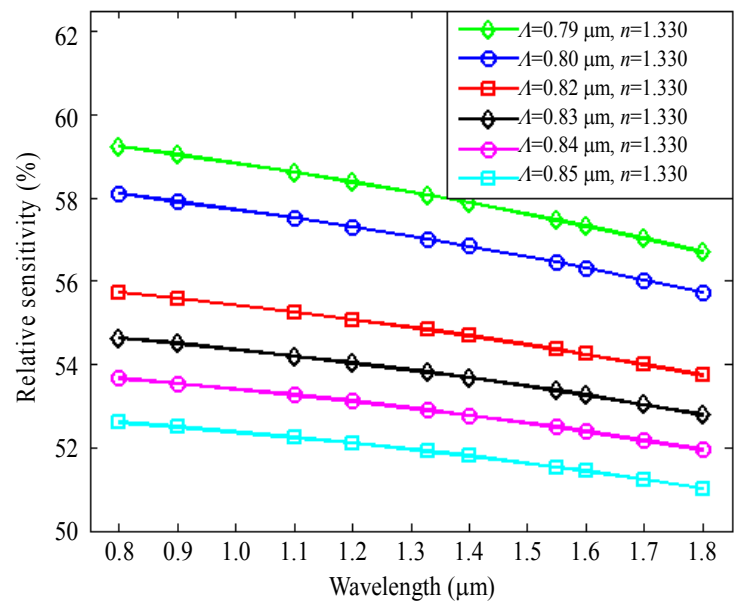

Fig. 10 Variation of the relative sensitivity versus wavelength of the P-HPCF due to different values of core pitch $\Lambda$ when other parameters are remained same for $n=1.33$. 


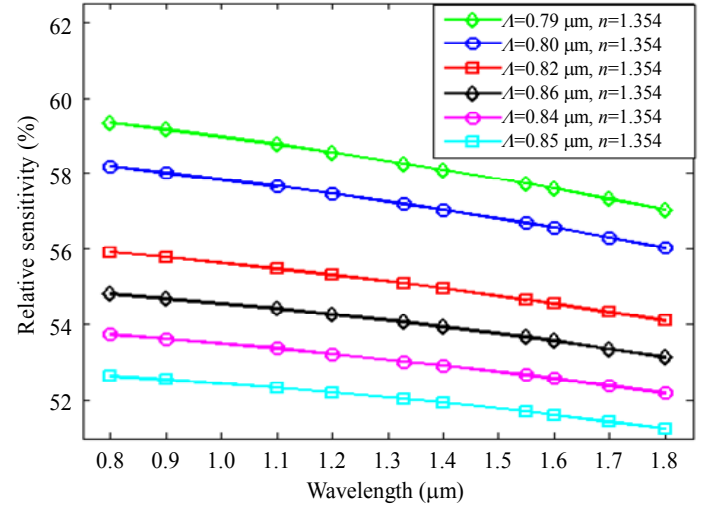

Fig. 11 Variation of the relative sensitivity versus wavelength of the P-HPCF due to different values of core pitch $\Lambda$ when other parameters are remained same for $n=1.354$.

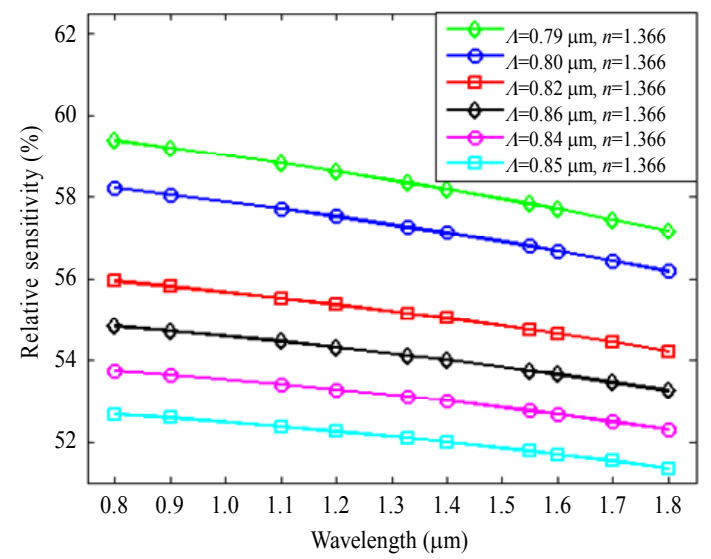

Fig. 12 Variation of the relative sensitivity versus wavelength of the P-HPCF due to different values of core pitch $\Lambda$ when other parameters are remained the same for $n=1.366$.

Figure 10 indicates the impact on the relative sensitivity of proposed P-HPCF for distinctive values of core pitch $\Lambda$ when applied liquid is water $(n=1.330)$. The sensitivities of $58.06 \%, 57.00 \%$, $54.82 \%, \quad 53.80 \%, \quad 52.90 \%, \quad$ and $\quad 51.90 \%$ correspondingly have been attained due to $\Lambda=$ $0.79 \mu \mathrm{m}, 0.80 \mu \mathrm{m}, 0.82 \mu \mathrm{m}, 0.83 \mu \mathrm{m}, 0.84 \mu \mathrm{m}$, and $0.85 \mu \mathrm{m}$ at $1.33 \mu \mathrm{m}$ wavelength. In addition, the confinement losses of $4.32 \times 10^{-11} \mathrm{~dB} / \mathrm{m}$, $2.15 \times 10^{-10} \mathrm{~dB} / \mathrm{m}, 1.46 \times 10^{-10} \mathrm{~dB} / \mathrm{m}, 7.22 \times 10^{-10} \mathrm{~dB} / \mathrm{m}$, $5.96 \times 10^{-12} \mathrm{~dB} / \mathrm{m}$, and $3.12 \times 10^{-11} \mathrm{~dB} / \mathrm{m}$ accordingly have been gained for similar requirements.

After finishing all examinations from Figs. 10, 11 , and 12 , it has been revealed that the high relative sensitivity and low confinement loss are achieved for $\Lambda=0.79 \mu \mathrm{m}$ due to applying three chemicals like water $(n=1.330)$, ethanol $(n=1.354)$, and benzene
( $n=1.366)$ at $1.33 \mu \mathrm{m}$ wavelength. To evade cost and complexity during the fabrication process, $\Lambda=$ $0.80 \mu \mathrm{m}$ is selected as the optimum pitch value of the core vicinity.

In a selective manner, filling the air holes of photonic crystal fiber by analytes is referred as a dare job. The air cavities of PCF in either the core or cladding region can be satiated by distinctive analytes with the progress of nanotechnology. A number of technical skills have been reported by the researchers. In 2004, Huang et al. [47] proposed a unique technique to fill the micro structured air cavities of both the core and cladding vicinities with distinctive analytes which may be accustomed to reveal the functionality of the PCF applications. This filling adaptation takes place by compelling the ultraviolet-curable polymer within the PCF. Zhang et al. [48] suggested a method for practical exhibition and speculate pretending of a liquid filled core based PCF to improve the sensing application. All investigated results in Table 1 have been manifestly indicated that the relative sensitivity and confinement loss are slightly varied with the optimum parameters after all modifications. So we confirmed that no global effects can influence the reported structure afterwards the fabrication process.

We have also examined the optimum parameters of $\pm 1 \%$ and $\pm 2 \%$ replacement as global parameters of the proposed P-HPCF. Comparison of relative sensitivity and confinement loss among the optimum parameters and the change in global parameters for water $(n=1.330)$, ethanol $(n=1.354)$, and benzene $(n=1.366)$ at $\lambda=1.33 \mu \mathrm{m}$ has been revealed in Table 1 . Table 2 demonstrates the comparison of the effective area and nonlinear coefficient of the optimum PHPCF structure for three applied liquids such as water, ethanol, and benzene at $\lambda=1.33 \mu \mathrm{m}$.

Several numbers of guiding attributes like frequency, propagation constant, total electric energy, total magnetic energy, and wave number in the free space of the proposed P-HPCF have been shown in Table 3. After completing the calculation process, it has been seen that propagation constant, total 
electric energy, and total magnetic energy are changed with different refractive index chemicals such as water $(n=1.330)$, ethanol $(n=1.354)$, and benzene $(n=1.366)$ except frequency and wave number in the free space.

Table 1 Comparison among the optimum parameters and the change in global parameters at $\lambda=1.33 \mu \mathrm{m}$.

\begin{tabular}{|c|c|c|c|c|c|c|}
\hline \multirow{2}{*}{$\begin{array}{l}\text { Change in global } \\
\text { parameters }(\%)\end{array}$} & \multicolumn{3}{|c|}{ Relative sensitivity (\%) } & \multicolumn{3}{|c|}{ Confinement loss $(\mathrm{dB} / \mathrm{m})$} \\
\hline & $n=1.33$ & $n=1.35$ & $n=1.366$ & $n=1.33$ & $n=1.354$ & $n=1.366$ \\
\hline$+2 \%$ & 57.99 & 57.24 & 57.32 & $6.84 \times 10^{-}$ & $2.01 \times 10^{-10}$ & $2.84 \times 10^{-10}$ \\
\hline$+1 \%$ & 56.93 & 57.21 & 58.16 & $1.21 \times 10^{-9}$ & $3.42 \times 10^{-10}$ & $8.16 \times 10^{-11}$ \\
\hline Optimum & 57.00 & 57.18 & 57.27 & $2.15 \times 10^{-10}$ & $1.11 \times 10^{-11}$ & $1.97 \times 10^{-11}$ \\
\hline$-1 \%$ & 56.87 & 57.16 & 57.24 & $1.22 \times 10^{-1}$ & $8.31 \times 10^{-10}$ & $1.36 \times 10^{-10}$ \\
\hline$-2 \%$ & 57.79 & 57.13 & 47.79 & $3.98 \times 10^{-9}$ & $1.90 \times 10^{-11}$ & $3.98 \times 10^{-9}$ \\
\hline
\end{tabular}

Table 2 Numerical values of effective area and nonlinear coefficient of the optimum P-HPCF structure at $\lambda=1.33 \mu \mathrm{m}$.

\begin{tabular}{ccc}
\hline Chemical $(n)$ & Effective area $\left(\mu \mathrm{m}^{2}\right)$ & Nonlinear coefficient $\left(\mathrm{W}^{-1} \mathrm{~km}^{-1}\right)$ \\
\hline Water $(n=1.330)$ & 11.99 & 11.82 \\
Ethanol $(n=1.354)$ & 11.85 & 11.95 \\
Benzene $(n=1.366)$ & 11.78 & 12.02 \\
\hline
\end{tabular}

Table 3 Different guiding values of the optimum P-HPCF structure at $\lambda=1.33 \mu \mathrm{m}$.

\begin{tabular}{cccc}
\hline Investigation parameters & Water & Ethanol & Benzene \\
\hline Frequency $(\mathrm{Hz})$ & $2.25 \times 10^{14}$ & $2.25 \times 10^{14}$ & $2.25 \times 10^{14}$ \\
Propagation constant $(\mathrm{rad} / \mathrm{m})$ & $6.21 \times 10^{6}$ & $6.32 \times 10^{6}$ & $6.38 \times 10^{6}$ \\
Total electric energy $(\mathrm{J})$ & $1.67 \times 10^{-7}$ & $1.74 \times 10^{-7}$ & $1.77 \times 10^{-7}$ \\
Total magnetic energy $(\mathrm{J})$ & $1.67 \times 10^{-7}$ & $1.74 \times 10^{-7}$ & $1.77 \times 10^{-7}$ \\
Wave number in free space $(\mathrm{rad} / \mathrm{m})$ & $4.72 \times 10^{6}$ & $4.72 \times 10^{6}$ & $4.72 \times 10^{6}$ \\
\hline
\end{tabular}

The comparative performance analysis between the prior PCFs and proposed P-HPCF has been listed in Table 4. Comparing with the prior PCFs, our proposed PCF depicts upward relative sensitivity and low confinement for ethanol $(n=1.354)$ analytes. The proposed P-HPCF is nobly improved the relative sensitivity approximately 2.41 , 1.30 , and 1.16 times compared with the previous PCFs [34], [35], and [10] respectively as well as diminishing the confinement loss from [34] and [10].

Table 4 Comparison of simulated result and structural shape among proposed P-HPCF and prior PCFs at $\lambda=1.33 \mu \mathrm{m}$ for $n=1.354$.

\begin{tabular}{|c|c|c|c|c|c|}
\hline \multirow{2}{*}{ PCFs } & \multirow{2}{*}{ Sen. $(\%)$} & \multirow{2}{*}{$\begin{array}{l}\text { Con. loss } \\
(\mathrm{dB} / \mathrm{m})\end{array}$} & \multirow{2}{*}{$\begin{array}{c}\text { No. of } \\
\text { ring }\end{array}$} & \multicolumn{2}{|c|}{ Structural shape } \\
\hline & & & & Core & Cladding \\
\hline $\begin{array}{c}\text { Prior } \\
\mathrm{PCF}_{1}[34]\end{array}$ & 23.75 & $2.40 \times 10^{-4}$ & 3 & Elliptical holes & $\begin{array}{c}\text { Circular holes } \\
\text { in octagonal }\end{array}$ \\
\hline $\begin{array}{c}\text { Prior } \\
\mathrm{PCF}_{2}[35]\end{array}$ & 46.87 & $2.28 \times 10^{-14}$ & 5 & Circular holes & $\begin{array}{c}\text { Circular holes } \\
\text { in octagonal }\end{array}$ \\
\hline $\begin{array}{c}\text { Prior } \\
\mathrm{PCF}_{3}[10]\end{array}$ & 49.17 & $2.75 \times 10^{-10}$ & 3 & Elliptical holes & $\begin{array}{l}\text { Circular holes } \\
\text { in circular }\end{array}$ \\
\hline Pro. PCF & 57.18 & $1.11 \times 10^{-11}$ & 5 & $\begin{array}{c}\text { Elliptical holes } \\
\text { in porous }\end{array}$ & $\begin{array}{l}\text { Circular holes } \\
\text { in hexagonal }\end{array}$ \\
\hline
\end{tabular}

The fabrication procedure is a significant topic for all proposed photonic crystal fibers. So the fabrication process of the proposed P-HPCF may be not comfortable. The proposed P-HPCF includes two different types of holes in cladding regions. Due to the technological progress in the fabrication of PCFs, our reported structure can be feasibly fabricated. Various fabrication techniques have been advanced for micro structured PCF like extrusion [49], stack and draw [50], drilling [51], and sol-gel casting [52]. Extrusion method contributes exemption in design. To utilize this method, material losses are extremely high for soft glasses, which is regarded as key drawback of this technique. The drilling technique allows fitting both the holes size and spacing. But circular shape fiber is restricted to drilling technique. In 2012, Hamzaoui et al. [52] fabricated an ionic copper-doped micro structured optical fiber by a new fabrication method which is renowned as a sol-gel technique. This method provides the freedom to modify air holes size, shape, and pitches. So, the proposed P-HPCF will be successfully fabricated by sol-gel casting technique.

\section{Conclusions}

A micro structured porous cored hexagonal photonic crystal fiber (P-HPCF) formed by five layered cladding embracing circular air holes and two layered porous coredwith elliptical air holes is reported in this paper. The proposed P-HPCF is accustomed as a chemical sensor. The FEM and PML circular boundary conditions are enforced for numerically investigated variation of effects on propagation characteristics of the proposed P-HPCF. All examined outcomes show that the proposed PHPCF exposes decent sensitivity and lower confinement loss compared with the prior PCFs at a wide scope of wavelength from $0.8 \mu \mathrm{m}$ to $1.8 \mu \mathrm{m}$. The proposed PCF reveals the relative sensitivity $57.00 \%, 57.18 \%$, and $57.27 \%$ correspondingly for water $(n=1.33)$, ethanol $(n=1.354)$, and benzene $(n=1.366)$ analytes. In addition, the confinement 
losses of $2.15 \times 10^{-10} \mathrm{~dB} / \mathrm{m}, 1.11 \times 10^{-11} \mathrm{~dB} / \mathrm{m}$, and $1.97 \times 10^{-11} \mathrm{~dB} / \mathrm{m}$ are gained for the same analytes. So it is apparent that the proposed P-HPCF based chemical sensor exhibits novel execution in industrial security intention at the lower band.

Open Access This article is distributed under the terms of the Creative Commons Attribution 4.0 International License (http://creativecommons.org/licenses/by/4.0/), which permits unrestricted use, distribution, and reproduction in any medium, provided you give appropriate credit to the original author(s) and the source, provide a link to the Creative Commons license, and indicate if changes were made.

\section{References}

[1] P. Russell, "Photonic crystal fibers," Science, 2003, 23(299): 358-362.

[2] H. Ademgil, "Highly sensitive octagonal photonic crystal fiber based sensor," Optik-International Journal for Light and Electron Optics, 2014, 125(20): 6274-6278.

[3] J. C. Knight, T. A. Birks, P. S. J. Russell, and D. M. Atkin, "All-silica single-mode optical fiber with photonic crystal cladding," Optics Letters, 1996, 21(19): 1547-1549.

[4] Y. L. Hoo, W. Jin, H. L. Ho, and D. N. Wang, "Measurement of gas diffusion coefficient using photonic crystal fiber," IEEE Photonics Technology Letters, 2003, 15(10): 1434-1436.

[5] M. Deng, C. Huang, D. Liu, W. Jin, and T. Zhu, “All fiber magnetic field sensor with ferrofluid-filled tapered microstructured optical fiber interferometer," Optics Express, 2015, 23(16): 20668-20674.

[6] J. M. Fini, "Microstructure fibres for optical sensing in gases and liquids," Measurement Science and Technology, 2004, 15(6): 1120-1128.

[7] J. C. Knight, T. A. Birks, P. S. J. Russell, and D. M. Atkin, "All-silica single-mode optical fiber with photonic crystal cladding," Optics Letters, 1996, 21(19): 1547-1549.

[8] S. M. A. Razzak, Y. Namihira, F. Begum, S. Kaijage, N. H. Hai, and N. Zou, "Design of a decagonal photonic crystal fiber for ultra-flattened chromatic dispersion," IEICE Transactions on Electronics, 2007, 90(11): 2141-2145.

[9] Y. Hou, F. Fan, Z. W. Jiang, X. H. Wang, and S. J. Chang, "Highly birefringent polymer terahertz fiber with honeycomb cladding," Optik-International Journal for Light and Electron Optics, 2013, 124(7): 3095-3098.
[10] S. Asaduzzaman, K. Ahmed, T. Bhuiyan, and T. Farah, "Hybrid photonic crystal fiber in chemical sensing," SpringerPlus, 2016, 5(1): 1-11.

[11] M. Morshed, M. I. Hasan, and S. M. A. Razzak, "Enhancement of the sensitivity of gas sensor based on microstructure optical fiber," Photonic Sensors, 2015, 5(4): 312-320.

[12] M. S. Habib, M. S. Habib, S. M. A. Razzak, and M. A. Hossain, "Proposal for highly birefringent broadband dispersion compensating octagonal photonic crystal fiber," Optical Fiber Technology, 2013, 19(5): 461-467.

[13] M. S. Habib, M. S. Habib, M. A. Hasan, and S. M. A. Razzak, "Single mode ultra-flat high negative residual dispersion compensating photonic crystal fiber," Optical Fiber Technology, 2014, 20(4): 328332.

[14] F. Begum, Y. Namihira, S. M. A. Razzak, S. Kaijage, N. H. Hai, T. Kinjo, et al., "Design and analysis of novel highly nonlinear photonic crystal fibers with ultra-flattened chromatic dispersion," Optics Communications, 2009, 282(7): 1416-1421.

[15]M. F. H. Arif, K. Ahmed, S. Asaduzzaman, and M. A. K. Azad, "Design and optimization of photonic crystal fiber for liquid sensing applications," Photonic Sensors, 2016, 6(3): 279-288.

[16] K. Nozaki, T. Tanabe, A. Shinya, S. Matsuo, T. Sato, H. Taniyama, et al., "Sub-femtojoule all-optical switching using a photonic-crystal nanocavity," Nature Photonics, 2010, 4(7): 477-483.

[17] J. M. Brosi, C. Koos, L. C. Andreani, M. Waldow, J. Leuthold, and W. Freude, "High-speed low-voltage electro-optic modulator with a polymer-infiltrated silicon photonic crystal waveguide," Optics Express, 2008, 16(6): 4177-4191.

[18] Y. Gao, R. J. Shiue, X. Gan, L. Li, C. Peng, I. Meric, et al., "High-speed electro-optic modulator integrated with graphene-boron nitride heterostructure and photonic crystal nanocavity," Nano Letters, 2015, 15(3): 2001-2005.

[19] D. Chen, "Stable multi-wavelength erbium-doped fiber laser based on a photonic crystal fiber Sagnac loop filter," Laser Physics Letters, 2007, 4(6): 437 439.

[20] H. Xuan, J. Ma, and W. Jin, "Polarization converters in highly birefringent microfibers," Optics Express, 2014, 22(3): 3648-3660.

[21]F. Benabid, F. Couny, J. C. Knight, T. A. Birks, and P. S. J. Russell, "Compact, stable and efficient all-fiber gas cells using hollow-core photonic crystal fibers," Nature, 2005, 434(7032): 488-491.

[22] J. M. Dudley, G. Genty, and S. Coen, "Supercontinuum generation in photonic crystal fiber," Reviews of Modern Physics, 2006, 78(4): 
1135-1184.

[23] K. Mileńko, D. J. J. Hu, P. P. Shum, T. Zhang, J. L. Lim, Y. Wang, et al., "Photonic crystal fiber tip interferometer for refractive index sensing," Optics Letters, 2012, 37(8): 1373-1375.

[24] T. Larsen, A. Bjarklev, D. Hermann, and J. Broeng, "Optical devices based on liquid crystal photonic bandgap fibers," Optics Express, 2003, 11(20): 2589-2596.

[25] H. Y. Fu, H. Y. Tam, L. Y. Shao, X. Dong, P. K. A. Wai, C. Lu, et al., "Pressure sensor realized with polarization-maintaining photonic crystal fiber-based Sagnac interferometer," Applied Optics, 2008, 47(15): 2835-2839.

[26]C. Zhao, X. Yang, C. Lu, W. Jin, and M. S. Demokan, "Temperature-insensitive interferometer using a highly birefringent photonic crystal fiber loop mirror," IEEE Photonics Technology Letters, 2004, 16(11): 2535-2537.

[27] M. Eichenfield, J. Chan, R. M. Camacho, K. J. Vahala, and O. Painter, "Optomechanical crystals," Nature, 2009, 462(7269): 78-82.

[28] K. Lee and S. A. Asher, "Photonic crystal chemical sensors: PH and ionic strength," Journal of the American Chemical Society, 2000, 122(39): 95349537.

[29] N. Skivesen, A. Têtu, M. Kristensen, J. Kjems, L. H. Frandsen, and P. I. Borel, "Photonic-crystal waveguide biosensor," Optics Express, 2007, 15(6): 3169-3176.

[30] L. D. Bonifacio, D. P. Puzzo, S. Breslav, B. M. Willey, A. McGeer, and G. A. Ozin, "Towards the photonic nose: a novel platform for molecule and bacteria identification," Advanced Materials, 2009, 22(12): 1351-1354.

[31] A. M. R. Pinto and M. Lopez-Amo, "Photonic crystal fibers for sensing applications," Journal of Sensors, 2012, 2012: 1-21.

[32] T. P. Hansen, J. Broeng, S. E. Libori, E. Knudsen, A. Bjarklev, J. R. Jensen, et al., "Highly birefringent index-guiding photonic crystal fibers," IEEE Photonics Technology Letters, 2001, 13(6): 588-590.

[33] S. Olyaee, M. Seifouri, A. Nikoosohbat, and M. S. E. Abadi, "Low nonlinear effects index-guiding nanostructured photonic crystal fiber," International Journal of Chemical, Nuclear, Materials and Metallurgical Engineering, 2015, 9(2): 253-257.

[34] H. Ademgil and S. Haxha, "PCF based sensor with high sensitivity, high birefringence and low confinement losses for liquid analyte sensing applications," Sensors, 2015, 15(12): 31833-31842.

[35] K. Ahmed and M. Morshed, "Design and numerical analysis of microstructured-core octagonal photonic crystal fiber for sensing applications," Sensing and
Bio-Sensing Research, 2016, 7: 1-6.

[36]A. N. Z. Rashed, E. N. A. E. G. Mohamed, S. A. E. R. S. Hanafy, and M. H. Aly, "A comparative study of the performance of graded index perfluorinated plastic and alumino silicate optical fibers in internal optical interconnections," Optik-International Journal for Light and Electron Optics, 2016, 127(20): 9259-9263.

[37] S. E. Kim, B. H. Kim, C. G. Lee, S. Lee, K. Oh, and C. S. Kee, "Elliptical defected core photonic crystal fiber with high birefringence and negative flattened dispersion," Optics Express, 2012, 20(2): 1385-1391.

[38] N. Luan and J. Yao, "Surface plasmon resonance sensor based on exposed-core microstructured optical fiber placed with a silver wire," IEEE Photonics Journal, 2016, 8(1): 1-8.

[39] S. Haxha, A. Teyeb, F. A. Malek, E. K. Akowuah, and I. Dayoub, "Design of environmental biosensor based on photonic crystal fiber with bends using finite element method," Optics and Photonics Journal, 2015, 05(3): 69-78.

[40] B. T. Kuhlmey, B. J. Eggleton, and D. K. C. Wu, "Fluid-filled solid-core photonic bandgap fibers," Journal of Lightwave Technology, 2009, 27(11): 1617-1630.

[41]M. Vieweg, T. Gissibl, S. Pricking, B. T. Kuhlmey, D. C. Wu, B. J. Eggleton, et al., "Ultrafast nonlinear optofluidics in selectively liquid-filled photonic crystal fibers," Optics Express, 2010, 18(24): 2523225240.

[42] S. Asaduzzaman and K. Ahmed, "Proposal of a gas sensor with high sensitivity, birefringence and nonlinearity for air pollution monitoring," Sensing and Bio-Sensing Research, 2016, 10: 20-26.

[43] F. Shi, G. Zhou, D. Li, L. Peng, Z. Hou, and C. Xia, "Surface plasmon mode coupling in photonic crystal fiber symmetrically filled with $\mathrm{Ag} / \mathrm{Au}$ alloy wires," Plasmonics, 2014, 10(2): 335-340.

[44] K. Kaneshima, "Numerical investigation of octagonal Photonic crystal fibers with strong confinement field," IEICE Transactions on Electronics, 2006, 89(6): 830-837.

[45] T. Matsui, J. Zhou, K. Nakajima, and I. Sankawa, "Dispersion-flattened photonic crystal fiber with large effective area and low confinement loss," Journal of Lightwave Technology, 2005, 23(12): 4178-4183.

[46] T. Sato, S. Makino, Y. Ishizaka, T. Fujisawa, and K. Saitoh, "A rigorous definition of nonlinear parameter ${ }_{1} \Gamma$ and effective area $\mathrm{A}_{\text {eff }}$ for photonic crystal optical waveguides," Journal of the Optical Society of America B, 2015, 32(6): 653-657.

[47] Y. Huang, Y. Xu, and A. Yariv, "Fabrication of 
functional microstructured optical fibers through a selective-filling technique," Applied Physics Letters, 2004, 85(22): 5182-5184.

[48]Y. Zhang, C. Shi, C. Gu, L. Seballos, and J. Z. Zhang, "Liquid core photonic crystal fiber sensor based on surface enhanced Raman scattering," Applied Physics Letters, 2007, 90(19): 193504-1-193504-3.

[49] H. Ebendorff-Heidepriem, P. Petropoulos, S. Asimakis, V. Finazzi, R. Moore, K. Frampton, et al., "Bismuth glass holey fibers with high nonlinearity," Optics Express, 2004, 12(21): 5082-5087.

[50] J. Broeng, D. Mogilevstev, S. E. Barkou, and A.
Bjarklev, "Photonic crystal fibers: a new class of optical waveguides," Optical Fiber Technology, 1999, 5(3): 305-330.

[51] M. N. Petrovich, A. V. Brakel, F. Poletti, K. Mukasa, E. Austin, V. Finazzi, et al., "Micro structured fibers for sensing applications," Proc. SPIE, 2005, 6005: 1-15.

[52] H. H. El, Y. Ouerdane, L. Bigot, G. Bouwmans, B. Capoen, A. Boukenter, et al., "Sol-gel derived ionic copper-doped micro structured optical fiber: a potential selective ultraviolet radiation dosimeter," Optics Express, 2012, 20(28): 29751-29760. 